\title{
Effect of hyperbaric oxygenation therapy on post-concussion syndrome (Review)
}

\author{
YANG DONG ${ }^{1,2}$, XIAHUAHU $^{1}, \mathrm{TAO}^{\mathrm{W}} \mathrm{WU}^{3}$ and TONG $\mathrm{WANG}^{4}$ \\ ${ }^{1}$ Department of Rehabilitation Medicine, Hangzhou Hospital of Zhejiang CAPF, Hangzhou, Zhejiang $310016 ;{ }^{2}$ Institute of \\ Rehabilitation Medicine, Nanjing Medical University, Nanjing, Jiangsu 210029; ${ }^{3}$ Department of Rehabilitation Medicine, \\ Sir Run Run Shaw Hospital, College of Medicine, Zhejiang University, Hangzhou, Zhejiang 310016; ${ }^{4}$ Department of \\ Rehabilitation Medicine, The First Affiliated Hospital of Nanjing Medical University, Nanjing, Jiangsu 210029, P.R. China
}

Received December 25, 2016; Accepted July 11, 2017

DOI: $10.3892 /$ etm.2018.6463

\begin{abstract}
The present review evaluated the effect of hyperbaric oxygenation (HBO) therapy on post-concussion syndrome (PCS). Searches for publications from the earliest date possible up until the first week of 2016 were conducted using the electronic databases Cochrane, EBSCOhost, Embase, Ovid MEDLINE, PubMed and Web of Science. Additional trials were identified through reference list scanning. Randomized controlled trials assessing the effectiveness of HBO therapy in PCS were selected and tested for eligibility for inclusion in the present review. Two independent reviewers conducted data extraction and the Cochrane Collaboration's recommended method was used to assess the risk of bias in each study included. Review Manager 5.3 software was used for data synthesis and analysis and the standardized mean difference (SMD) or mean difference (MD) was estimated with a fixed or random effects model using a 95\% confidence interval (CI). A total of 127 articles were identified, 4 of which were eligible for final analysis. The meta-analysis identified no difference in the Rivermead Post-Concussion Symptoms Questionnaire ( $\mathrm{MD}=1.23$; 95\% CI, -3.47-5.94; $\left.\mathrm{P}>0.05 ; \mathrm{I}^{2}=35 \%\right)$ or Post-Traumatic Stress Disorder Checklist (PCL) scores $\left(\mathrm{SMD}=0.12 ; 95 \% \mathrm{CI},-0.31-0.54 ; \mathrm{P}>0.05 ; \mathrm{I}^{2}=0 \%\right)$ scores between groups receiving different oxygen doses. The differences in PCL scores (SMD $=-0.13,95 \%$ CI, -0.80-0.53; $\mathrm{P}>0.05 ; \mathrm{I}^{2}=63 \%$ ) and neurobehavioral symptoms ( $\mathrm{SMD}=-1.00$, $95 \%$ CI, $-2.58-0.58 ; \mathrm{P}>0.05 ; \mathrm{I}^{2}=92 \%$ ) between the $\mathrm{HBO}$ and
\end{abstract}

Correspondence to: Dr Tong Wang, Department of Rehabilitation Medicine, The First Affiliated Hospital of Nanjing Medical University, 300 Guangzhou Road, Nanjing, Jiangsu 210029, P.R. China

E-mail: wangtong60621@aliyun.com

Abbreviations: HBO, hyperbaric oxygenation; ATA, atmospheres absolute; RPQ, Rivermead Post-Concussion Symptoms Questionnaire; NSI, Neurobehavioral Symptom Inventory; PCL, Post-Traumatic Stress Disorder Checklist; EQ-5D, EuroQoL Group's 5-dimension questionnaire

Key words: post-concussion syndrome, hyperbaric oxygenation, review sham groups were not significant. The current study demonstrated that HBO therapy has no significant effect on PCS compared with the sham group. Therefore, it was determined that effective design and execution of a large clinical trial, which includes treatment, control and sham groups is required in the future.

\section{Contents}

1. Introduction

2. Methods

3. Study selection and participants

4. Clinical outcomes

5. Discussion

\section{Introduction}

Traumatic brain injury (TBI) is a major cause of death and disability that disproportionately affects young adults (1). The persistence of symptoms for $>3$ months following the onset of mild TBI is known as post-concussion syndrome (PCS). The incidence of PCS following the onset of TBI is $\sim 15 \%$ after 3 months and 3-5\% after 1 year (2). Common post-concussion symptoms include headaches, balance problems, sleep disturbance, fatigue, forgetfulness, poor concentration, irritability and anxiety (3). There are currently few established therapies available to treat patients with persistent PCS.

Hyperbaric oxygenation (HBO) therapy is currently used to treat acute and chronic ischemic injuries. HBO has well established theoretical underpinnings and is able to treat dive-related injuries, soft tissue injuries and carbon monoxide poisoning (4). The results of several studies have provided inconclusive evidence for the efficacy of HBO therapy in treating patients with PCS. Previous studies lacking control groups compared data pre- and post-HBO and found that $\mathrm{HBO}$ has a beneficial effect $(5,6)$. However, a selection of prospective randomized trials did not prove the therapeutic effectiveness of HBO in PCS following mild TBI $(7,8)$.

TBI is categorized into two phases: The primary insult and ensuing secondary reaction. A variable degree of irreversible 
primary damage to the neurological tissue occurs at the onset of injury (9). Secondary pathologies of TBI include ischemia, edema, hypoxia and other biochemical and inflammatory processes $(10,11)$. Local hypoxia and ischemia may lead to the initiation of neuronal cell death. The use of HBO to treat TBI is based on the fact that hypoxia may serve an important role in causing secondary injury (12).

Previous studies in animals have demonstrated that HBO may have beneficial effects on brain injury. HBO limits the growth of cerebral contusions (13), increases the contused hippocampus vascular density (14), decreases the extent of secondary cell death and reactive neuroinflammation (15), preserves mitochondrial integrity and inhibits the mitochondrial apoptotic pathway (16). As an adjunctive treatment for patients with TBI, HBO may reduce the risk of mortality and improve the final Glasgow Coma Scale score (17). However there is little evidence that the prognosis of survivors improves following $\operatorname{HBO}(18,19)$.

The present study conducted a systematic review and meta-analysis of the current literature to examine the benefit of HBO therapy in the treatment of patients with PCS. The current available clinical evidence was presented in order to provide a foundation for future research.

\section{Methods}

The present systematic review was conducted according to the Preferred Reporting Items for Systematic Reviews and Meta-Analyses guidelines (20) and was registered on the PROSPERO database (crd.york.ac.uk/PROSPERO; registration no. CRD 42016032620).

Study retrieval and screening. Studies were identified through by searching the Cochrane (cochranelibrary.com), EBSCOhost (search.ebscohost.com), Embase (embase.com/login), Ovid MEDLINE (ovidsp.ovid.com), PubMed (ncbi.nlm.nih. gov/pmc) and Web of Science (login.webofknowledge.com) databases. Database searches were limited to peer-reviewed scholarly journal articles published in English from inception up until the first week of 2016. Keywords, medical sub headings and an all fields search were conducted using the terms 'hyperbaric oxygenation' and 'post-concussion syndrome' to obtain articles meeting the eligibility criteria.

The results were analyzed independently by two reviewers. Three additional studies were identified from the reference lists of the retrieved studies, review articles and textbooks. All hits obtained with the search strategies were imported into EndNote version X7 (Clarivate Analytics, Philadelphia, PA, USA) and duplicates were subsequently removed.

The titles and abstracts of the remaining studies were screened by two reviewers independently to assess their eligibility. The full texts of potentially eligible studies were retrieved and assessed according to the inclusion criteria by the same two reviewers. Disagreements between the reviewers regarding the eligibility of titles/abstracts or full texts were resolved in a consensus meeting. In the case where consensus was not reached, a third reviewer was asked to make the final decision.

Eligibility criteria. The studies that satisfied all the following criteria were eligible for inclusion in the present review: i) Full-text articles published in a peer-reviewed scientific journal; ii) randomized controlled trials (RCTs) aimed at assessing the effectiveness of HBO in PCS; and iii) articles written in English. Exclusion criteria included studies that were not written in English, not RCTs or conducted in patients with mild brain injury who were not diagnosed with PCS.

Methodological quality assessment. The Cochrane Risk of Bias tool (21) was used to assess the methodological quality of the included studies in terms of sequence generation, allocation concealment, blinding, incomplete outcome data, selective outcome reporting and other sources of bias.

Data extraction. The following data were extracted from the full texts of the studies included in the present review: Author, year published, study design, population, sample size, patient age and sex, intervention and comparison, outcome and outcome administered time. Data were extracted by one reviewer and reviewed a second time by a different reviewer to ensure accuracy.

Statistical analysis. Review Manager 5.3 (The Nordic Cochrane Centre, The Cochrane Collaboration; ims.cochrane. org/revman) was used for data analysis. Pre- and post-intervention data were analyzed and compared. The weighted mean difference (MD) was pooled for continuous outcomes using the same measurement and the standardized mean difference (SMD) was calculated for continuous outcomes with different measurements. Statistical heterogeneity was detected using the Q statistic and $\mathrm{I}^{2}>50 \%$ indicated high heterogeneity. Using a $95 \%$ confidence interval (CI) a fixed effect model was used where there was no evidence of significant heterogeneity between studies and a random effects model when such heterogeneity was high. $\mathrm{P}<0.05$ was considered to indicate a statistically significant difference.

\section{Study selection and participants}

Study selection and characteristics. A total of 126 articles were identified through database searching and 43 of these were screened following the removal of duplicates (Fig. 1). These included 13 comments and reviews (22-34), 9 letters to the editor and their matching responses (35-43), two conference abstracts $(44,45)$, one paper on study design (2), one animal experiment (46) and one study on side effects (47). These studies were not RCTs and were therefore excluded. Concussion was not the focus of four of the studies screened (48-51) and an additional four studies $(5,6,19,52)$ were not RCTs. Therefore following the exclusion of the aforementioned studies, eight RCTs (4,7,53-57) were suitable for inclusion in the current review (Fig. 1). Four of these (7,53-55) reported different aspects of the same study and another two $(4,42)$ reported on the same trial; therefore these articles were evaluated together. A total of four articles were therefore used in the subsequent meta-analysis $(4,8,55,56)$. Characteristics of the included studies are presented in Table I.

Participants. A total of 238 patients were enrolled in the studies included in the present review. The majority of these were members of the military service $(4,8,55)$ apart from 


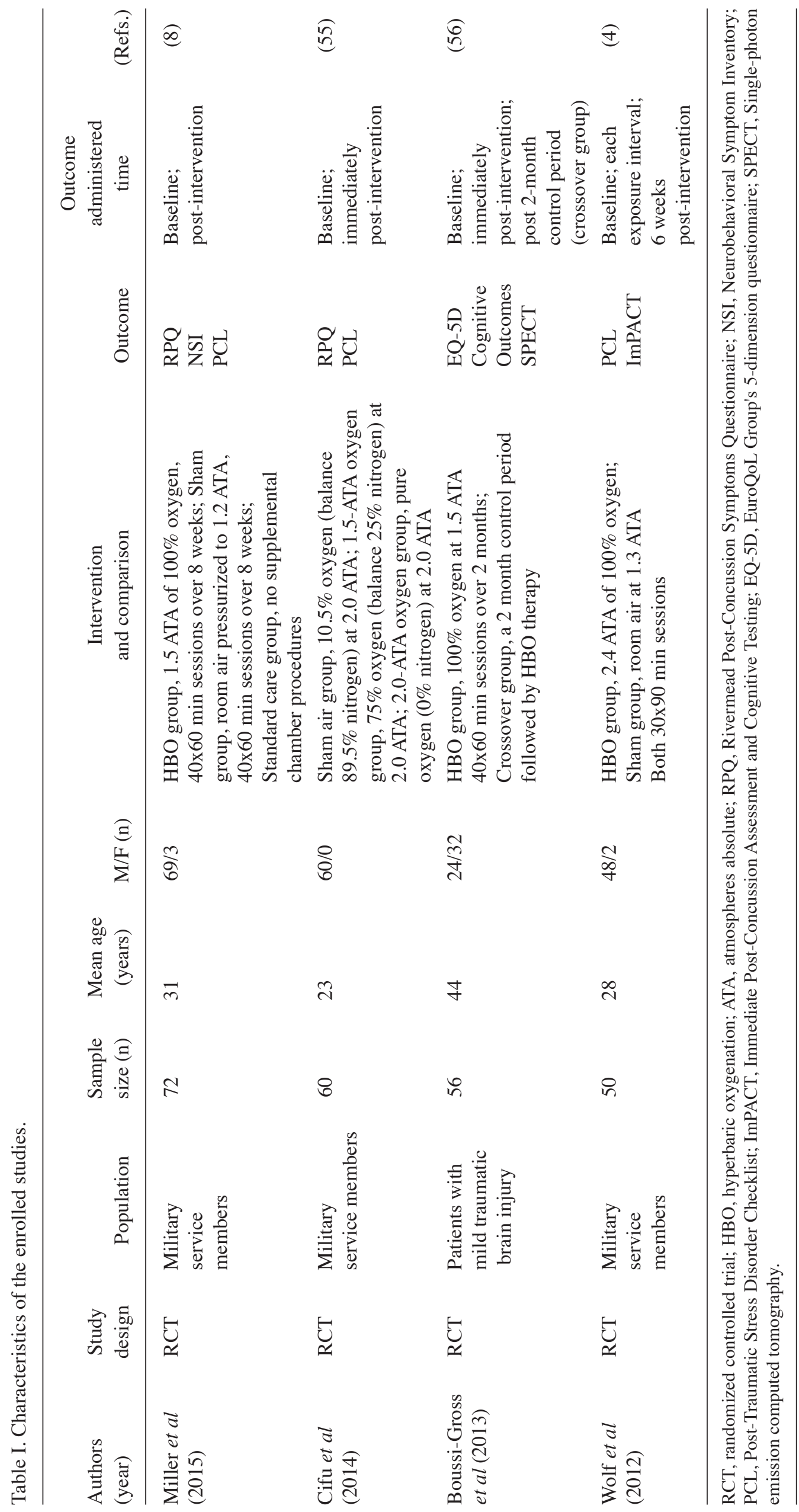




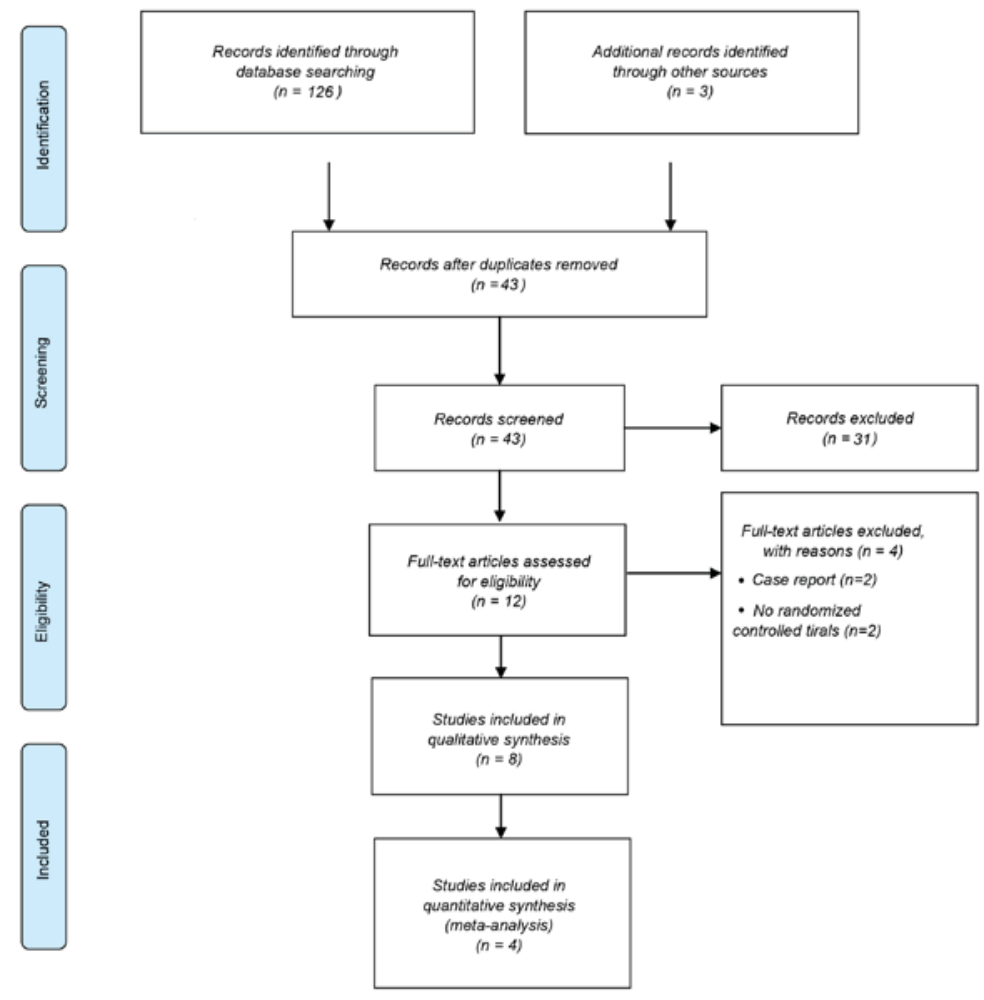

Figure 1. Review flowchart.

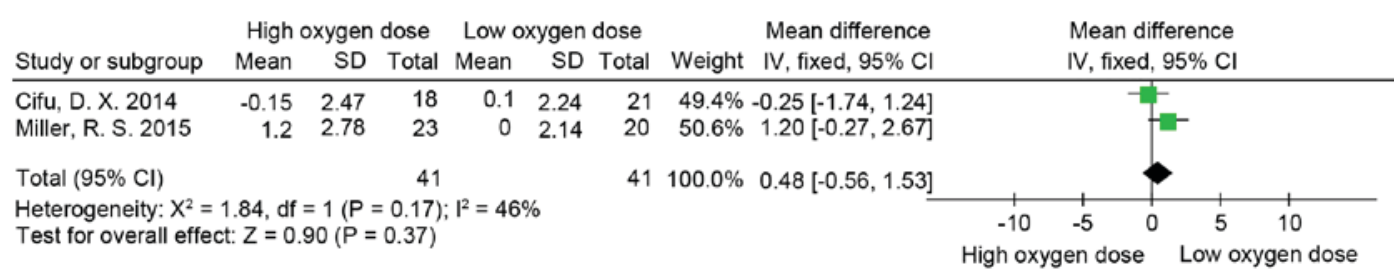

Figure 2. Rivermead Post-Concussion Symptoms Questionnaire-3 comparison between different oxygen dosage groups. SD, standard deviation; IV, inverse variance; CI, confidence interval; df, degrees of freedom.

the participants of one study, who were patients with mild, traumatic brain injury (56). The age range of all participants was 23-44 years and there were 201 males and 37 females.

Intervention. Trial designs varied among the studies included in the present review (Table I). A crossover control without a sham group was used in one study (56). Patients in the treatment group underwent $40 \mathrm{HBO}$ sessions for 60 min with $100 \%$ oxygen at 1.5 atmospheres absolute (ATA). Patients in the crossover group underwent the same HBO therapy following a 2 month control period of no treatment. HBO and sham groups were used in two of the other studies $(4,8)$ and one study also included a standard care group with no supplemental chamber procedures (8). In one study, patients in the HBO group underwent a series of 30 hyperbaric chamber compressions at 2.4 ATA with $100 \%$ oxygen, once each day for $90 \mathrm{~min}$ over an 8 week period, whereas participants in the sham group breathed air at 1.2-1.3 ATA (4). In the other study, patients in the HBO group underwent a series of 4060 min hyperbaric chamber compressions at 1.5 ATA with $100 \%$ oxygen over 8 weeks and participants in the sham group breathed air at 1.2 ATA (8). In a study by Cifu et al (55), subjects breathed
1 of 3 pre-assigned oxygen fractions for $60 \mathrm{~min}$, including $10.5 \%$ oxygen, $75 \%$ oxygen or $100 \%$ oxygen all at 2.0 ATA, resulting in an exposure to oxygen equivalent to breathing surface air, $100 \%$ oxygen at 1.5 ATA or $100 \%$ oxygen at 2.0 ATA, respectively.

\section{Clinical outcomes}

Rivermead post-concussion symptoms questionnaire (RPQ). RPQ (58) scores were measured in two of the trials included in the present review $(8,55)$. The psychometric properties of the RPQ suggest that it is most appropriately scored and analyzed using two subscales. These subscales consist of items 1-3, which constitute the RPQ-3 score and the remaining 13 items constitute the RPQ-13 score $(59,60)$. Following measurement of the individual doses of oxygen in partial pressures and concentration of oxygen multiplied by time for each treatment, the oxygen equivalent patients of the two studies were pooled into one group, thus generating two new groups: A high oxygen dose group and the low oxygen dose group. The difference in RPQ-3 (MD $=0.48 ; 95 \%$ CI, $-0.56-1.53 ; \mathrm{P}>0.05 ; \mathrm{I}^{2}=46 \%$; Fig. 2 ), RPQ-13 (MD=0.91; 95\% CI, -3.04-4.86; $\mathrm{P}>0.05 ; \mathrm{I}^{2}=20 \%$; 


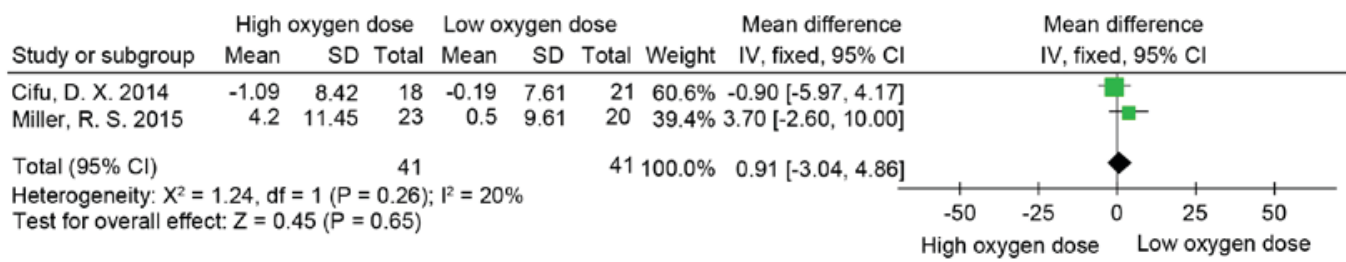

Figure 3. Rivermead Post-Concussion Symptoms Questionnaire-13 comparison between different oxygen dosage groups. SD, standard deviation; IV, inverse variance; CI, confidence interval; df, degrees of freedom.

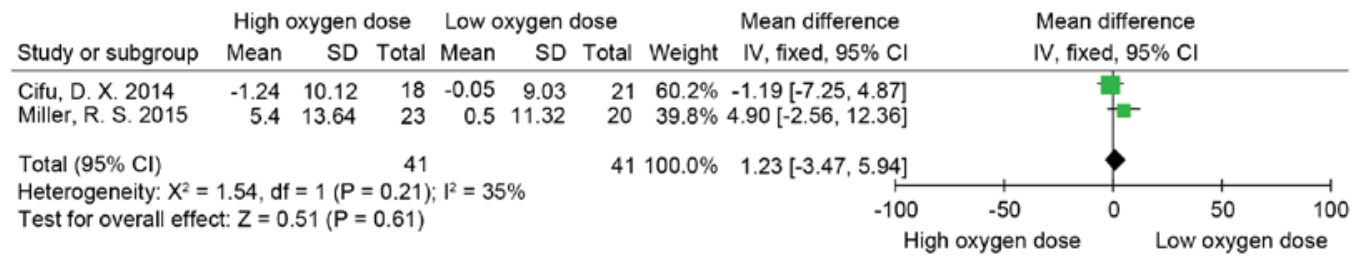

Figure 4. Rivermead Post-Concussion Symptoms Questionnaire-total comparison between different oxygen dosage groups. SD, standard deviation; IV, inverse variance; CI, confidence interval; df, degrees of freedom.

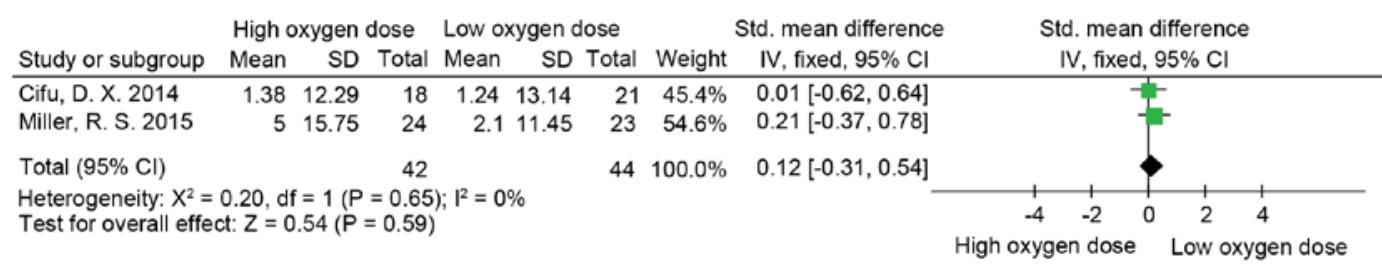

Figure 5. Post-traumatic stress disorder checklist comparison between different oxygen dosage groups. SD, standard deviation; IV, inverse variance; CI, confidence interval; df, degrees of freedom.

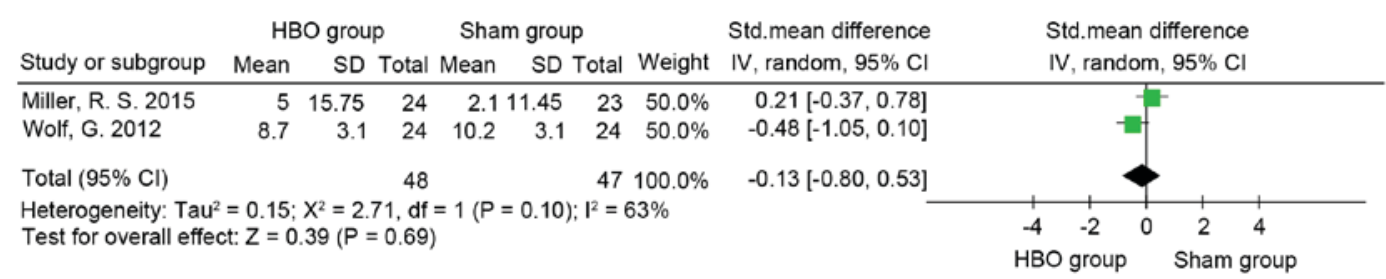

Figure 6. Post-traumatic stress disorder checklist comparison between $\mathrm{HBO}$ and sham groups. SD, standard deviation; IV, inverse variance; CI, confidence interval; df, degrees of freedom; HBO, hyperbaric oxygenation.

Fig. 3) and RPQ-total scores (MD=1.23; 95\% CI, -3.47-5.94; $\mathrm{P}>0.05 ; \mathrm{I}^{2}=35 \%$; Fig. 4) was not significant between the low and high oxygen dose groups.

Post-Traumatic Stress Disorder Checklist (PCL). A total of 3 out of 4 studies included PCL in their results $(4,8,55)$. Two $(4,52)$ used the military version $(60)$ and one $(8)$ used the civilian version (61). The results indicated that the difference between the high and low oxygen dose groups $(\mathrm{SMD}=0.12$; 95\% CI, -0.31-0.54; P>0.05; $\mathrm{I}^{2}=0 \%$; Fig. 5) and $\mathrm{HBO}$ and sham groups (SMD, $-0.13 ; 95 \% \mathrm{CI},-0.8$ to 0.53 ; $\mathrm{P}>0.05$; $\mathrm{I}^{2}=63 \%$; Fig. 6) was not significant.

Neurobehavioral symptom assessment. The neurobehavioral symptoms of participants were monitored in two trials $(4,8)$ using the Neurobehavioral Symptom Inventory (NSI) $(8,62)$ and Immediate Post-Concussion Assessment and Cognitive
Testing (4,63), respectively. The difference between the neurobehavioral symptoms of subjects in the $\mathrm{HBO}$ and sham groups was not significant $(\mathrm{SMD}=-1 ; 95 \% \mathrm{CI},-2.58-0.58$; $\mathrm{P}>0.05 ; \mathrm{I}^{2}=92 \%$; Fig. 7).

Health-related quality of life (QOL). Miller et al (8) demonstrated an improvement in health-related QOL outcomes, including physical functioning, bodily pain, social functioning and emotionality on the 36-Item Short Form Health Survey (SF-36) in the sham group compared with the HBO group and in the HBO group compared with the standard care group. QOL was evaluated in another trial using the EuroQuol five dimensions (EQ-5D) questionnaire (56). The EQ-5D questionnaire scores significantly improved following HBO therapy in the treated and crossover groups compared with the control group $(\mathrm{P}<0.05)$. However, no improvements were observed in the EQ-5D score in the crossover group following the control 


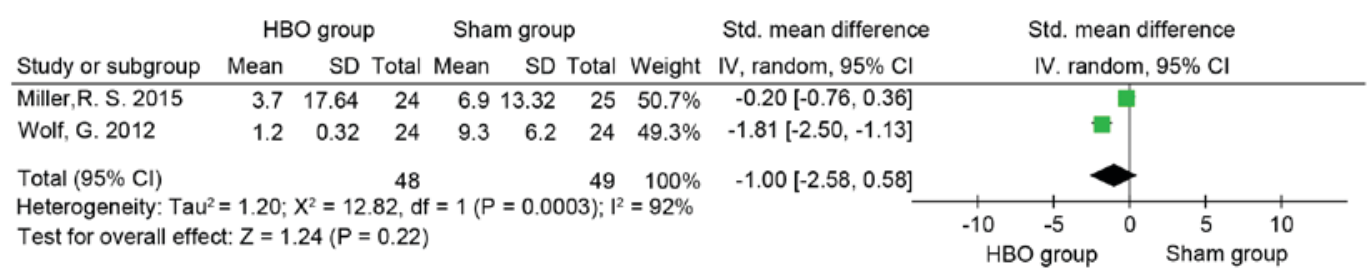

Figure 7. Neurobehavioral symptom comparison between $\mathrm{HBO}$ and sham groups. SD, standard deviation; IV, inverse variance; CI, confidence interval; df, degrees of freedom; HBO, hyperbaric oxygenation.

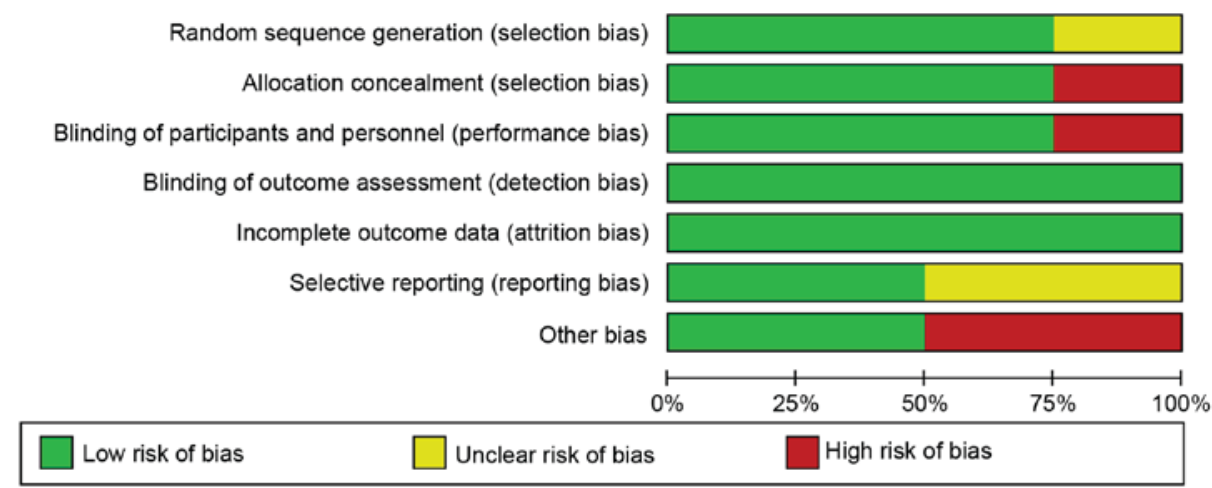

Figure 8. Risk-of-bias graph.

period. These results suggest that HBO therapy may improve QOL compared with the no supplemental chamber procedure, but not compared with the sham group. The standard deviation of SF-36 total score change was not available, thus a meta-analysis on QOL was not performed.

Cognitive function. There were insufficient data to conduct a meta-analysis on cognitive function. The crossover design study demonstrated significant improvements in cognitive function in the groups following HBO but no significant improvement following the control period (56). Single-photon emission computed tomography (SPECT) imaging also revealed elevated brain activity with cognitive improvements (56). Exposure to 1.5 or 2.0 ATA did not improve cognitive function compared with the sham air intervention in one study (7). Wolf et al (42) demonstrated that the difference in cognitive function between the sham and treatment groups undergoing HBO therapy at 2.4 ATA was not significant.

Quality and risk of bias. There was a low risk of bias in blinding outcome assessment and incomplete outcome data in the studies included in the present review (Fig. 8). However, the crossover study exhibited high risk regarding the blinding participants and personnel, as well as allocation concealment. A high risk of other bias was identified in two studies, including regarding the sham group as a control. The randomization method was unclear in 1 out of 4 studies. Therefore, the risk of bias among all studies was classed as medium due to selection, reporting and performance biases (Fig. 8).

\section{Discussion}

PCS is a term used to describe the complex and controversial physical, cognitive and emotional symptoms associated with mild brain injury. PCS persists for weeks or months in the majority of patients and $<25 \%$ of patients may experience prolonged PCS in which symptoms last for $>6$ months (64). These patients are at high risk of emotional and cognitive dysfunction in which they may be unable to perform ordinary daily activities and maintain work responsibilities, as well as normal social relationships $(65,66)$. Currently, there is limited evidence that multifaceted rehabilitation programs that include psychotherapy improve the management of persistent symptoms in PCS (67). Based on previous studies investigating other neurological conditions, it has been suggested that HBO therapy may be a potential treatment for chronic PCS $(5,6,52)$.

The results of the present systematic review identified no improvement in RPQ score or symptoms of PCS between low and high oxygen dose groups and no significant difference in the improvement of neurobehavioral symptoms between $\mathrm{HBO}$ and sham groups. Thus, there is no evidence that HBO therapy is effective at treating patients with PCS. However, HBO therapy is a combination of increased pressure and increased pressure of oxygen above ambient atmospheric pressure and the sham designs used in the studies included in the present review may not have tested an extensive enough range of pressurized air doses $(37,57)$.

The effects of air pressure have been investigated since the early 20th century; however, they have been more actively studied since the 1990s. The majority of studies performed so far have been cell culture experiments (57). A follow-up study by Mulkey et al (68) suggests that neuronal barosensitivity occurs at pressures of $100 \mathrm{mmHg}$ (1.13 ATA). Furthermore, it has been demonstrated that even a small increase in partial pressure to 1.05 ATA at an altitude of $402 \mathrm{~m}$ below sea level may induce noticeable physiological effects, such as improved pulmonary function and blood oxygen saturation (69-71). A room air pressure of 1.2 or 1.3 ATA may not be appropriate 
for sham controls, as this may lead to significant increases in tissue oxygenation (72). Thus, the use of $21 \%$ oxygen at 1.14-1.5 ATA in for sham controls in clinical trials as an alternative to observation or crossover controls may lead to false acceptance of the null hypothesis, due to the biological activity that occurs under these conditions (29). Consequently, studies that included a sham group were identified as having high bias $(4,7,8,55)$. The minimum pressure at which patients sense an increase in air pressure is 1.3 ATA. Controlled experiments testing the effects of $\mathrm{HBO}$ therapy must therefore ensure that pressure and oxygen concentrations are not above base levels in the control groups to meet the true definition of a sham (73). However, this may result in inherent ethical and logistic difficulties in handling the sham control in HBO trials.

Objective and precise assessment methods are another challenge in evaluating the efficacy of HBO therapy in patients with PCS. To the best of our knowledge, validated outcome measures for intervention trials in PCS have not yet been established. In the majority of the studies included in the present review, outcomes were evaluated using RPQ, PCL or NSI. All of these assessments are well established; however, they are all subjective performance evaluations (58,60-62). The RPQ has several limitations in its implementation and ability to accurately reflect test-taker experience (74). The interpretation and accuracy of the RPQ and other methods varies widely due to self-administration and the confounding variables involved due to its sensitivity to covariates, including subjective patient memory, social desirability, stress, personality factors and the willingness of patients to reveal problems (74). The studies included in the present review relied on self-administration assessments, which is a limitation. SPECT imaging was used in one randomized, crossover controlled trial and revealed elevated brain activity with cognitive improvements following HBO therapy in the treated and crossover groups (56). This is consistent with the results of previous studies $(5,6)$. Although the use of SPECT imaging may not be sensitive enough to detect abnormalities in patients with PCS, it is an objective assessment method that may provide evidence supporting the use of HBO or sham interventions and allows a greater refinement of HBO treatment for patients with PCS.

A study of HBO therapy used to treat sub-acute moderate to severe TBI at 2.0 ATA reported a 9\% seizure rate (75). However, serious side effects from HBO therapy are rare in patients with chronic and mild TBI and a previous study demonstrated that patients with TBI treated with HBO do not experience any marked side effects (76). Two trials in the current study reported that adverse events occurred during HBO therapy, which were equally distributed between the HBO and sham groups $(8,47)$ included in the present review. Serious adverse events, including pulmonary barotraumas, pulmonary edema or seizure were not observed.

There are several differences between blast-related and sports-related PCS. Patients with blast mild TBI have usually experienced two episodes of head trauma, often within sec of each other. The magnitude of head acceleration is stronger than that of a sports-related concussion and the entire body is exposed to the blast. Consequently, blast TBI is caused by multiple, interwoven mechanisms of systemic, local and cerebral responses to blast exposure (77). The majority of patients included in the present review were from the military, thus, the results are not representative of patients diagnosed with sports-related concussion.

Previous studies have demonstrated that there is an optimal therapeutic time frame for $\mathrm{HBO}$ treatment in neonatal rats with hypoxic-ischemic brain damage (78-80). However, to the best of our knowledge, the optimal timing of treatment for patients with brain injury remains unknown. Efrati and Ben-Jacob (23) suggested that HBO therapy may begin either at the degenerative or regenerative stages and is usually safe 1 month following acute injury. Subset analysis of isolated mild TBI demonstrated a trend toward harm from $\mathrm{HBO}$ at 2.4 ATA, suggesting that $\mathrm{HBO}$ at 2.4 ATA may actually have a negative impact on isolated mild TBI symptoms (81), however, the optimal effective doses of pressure and oxygen concentration in HBO therapy remain unclear, as does the optimal duration of treatment. The majority of the results of the studies included in the present review were obtained following 30-40 sessions of HBO therapy. It is hypothesized that additional sessions of HBO therapy may be beneficial (43) however, to the best of our knowledge, no data are available on the upper time limit at which no further improvements occur. The long-term effects of HBO therapy are not well studied. In the studies included in the current review, the time point for outcome assessment was usually $<1$ week following completion of treatment. One previous study identified that treating blast-related PCS 3 months following compression had no significant effect (53). Thus, further studies are required to develop understanding of the optimal number, duration and long-term effects of treatment sessions and the optimum time frame following injury onset for initiating HBO therapy.

Case reports and phase I clinical trials have demonstrated that $\mathrm{HBO}$ is an effective therapy for correctly diagnosed PCS $(5,6)$ however, the results of RCTs included in the current review were not consistent with this. HBO therapy may improve the symptoms of patients with increased levels of oxygen concentration and pressure. The results of the current meta-analysis indicated that increased doses of oxygen had no effect on PCS. The efficacy of pressurization remains elusive, as the symptoms of patients in the $\mathrm{HBO}$ and sham groups were improved with no difference in improvements observed between them. It is therefore essential to develop a sham that controls for pressurization or oxygen concentration separately in RCTs.

Although it remains unknown whether the symptoms of PCS improve following HBO therapy, it has been demonstrated that HBO therapy does not cause any serious side effects; thus, it has been recommended that patients with PCS should undergo HBO therapy, until future studies are completed. However, it should be noted that HBO treatment is costly and potentially dangerous thus, its use must be evidence-based (82). The use of genuine sham controls in studies on HBO therapy is not feasible or cost effective and the optimal therapeutic window and oxygen dose of HBO therapy remain unknown. Therefore future studies should be conducted on a large scale or in cohorts to produce more useful results. The approval of HBO therapy on a tentative basis would allow for studies to be conducted on a large patient population.

There were several limitations of the current meta-analysis. Although a comprehensive search of six databases was conducted, only four studies consisting of 238 patients in total were included in the present systematic review. The small 
number of included studies limited the statistical power of detection. Additionally, the sample set of patients used is not representative of patients with sport-related PCS due to the small number of studies included, which primarily included members of the military that had experienced blast-induced PCS. A comparison of all groups was not conducted due to the heterogeneity between different trials. In addition, the study design of the sham group may lead to high bias. Therefore, more rigorous reviews are required to assess the effects and safety of HBO therapy in patients with PCS.

In conclusion, the present systematic review demonstrated that HBO therapy was not associated with significant improvements in patients with PCS. Large scale observation or cohort studies are required to provide information for the design and execution of a large clinical trial consisting of proper treatment, control and sham groups. This future trial may subsequently provide the evidence required for the efficacy of HBO therapy in the treatment of patients with PCS.

\section{Acknowledgements}

Not applicable.

\section{Funding}

No funding was received.

\section{Availability of data and materials}

All data generated or analyzed during this study are included in this published article.

\section{Author's contributions}

ToW made substantial contributions to the conception and design of the study. YD and XHH searched and analyzed the data independently. In the case where consensus was not reached, TaW was asked to make the final decision and interpret the data.

\section{Ethics approval and consent to participate}

Not applicable.

\section{Patient consent for publication}

Not applicable.

\section{Competing interests}

All authors declare no conflicts of interest.

\section{References}

1. Hyder AA, Wunderlich CA, Puvanachandra P, Gururaj G and Kobusingye OC: The impact of traumatic brain injuries: A global perspective. NeuroRehabilitation 22: 341-353, 2007.

2. Weaver LK, Cifu D, Hart B, Wolf G and Miller S: Hyperbaric oxygen for post-concussion syndrome: Design of department of defense clinical trials. Undersea Hyperb Med 39: 807-814, 2012.

3. King NS: Post-concussion syndrome: Clarity amid the controversy? Br J Psychiatry 183: 276-278, 2003.
4. Wolf G, Cifu D, Baugh L, Carne W and Profenna L: The effect of hyperbaric oxygen on symptoms after mild traumatic brain injury. J Neurotrauma 29: 2606-2612, 2012.

5. Harch PG, Fogarty EF, Staab PK and Van Meter K: Low pressure hyperbaric oxygen therapy and SPECT brain imaging in the treatment of blast-induced chronic traumatic brain injury (post-concussion syndrome) and post traumatic stress disorder: A case report. Cases J 2: 6538, 2009.

6. Harch PG, Andrews SR, Fogarty EF, Amen D, Pezzullo JC, Lucarini J, Aubrey C, Taylor DV, Staab PK and Van Meter KW: A phase I study of low-pressure hyperbaric oxygen therapy for blast-induced post-concussion syndrome and post-traumatic stress disorder. J Neurotrauma 29: 168-185, 2012.

7. Walker WC, Franke LM, Cifu DX and Hart BB: Randomized, Sham-Controlled, feasibility trial of hyperbaric oxygen for service members with postconcussion syndrome: Cognitive and psychomotor outcomes 1 week postintervention. Neurorehabil Neural Repair 28: 420-432, 2013.

8. Miller RS, Weaver LK, Bahraini N, Churchill S, Price RC, Skiba V, Caviness J, Mooney S, Hetzell B, Liu J, et al: Effects of hyperbaric oxygen on symptoms and quality of life among service members with persistent postconcussion symptoms: A randomized clinical trial. JAMA Intern Med 175: 43-52, 2015.

9. Loane DJ, Stoica BA and Faden AI: Neuroprotection for traumatic brain injury. Handb Clin Neurol 127: 343-366, 2015.

10. Fiskum G: Mitochondrial participation in ischemic and traumatic neural cell death. J Neurotrauma 17: 843-855, 2000.

11. Roth TL, Nayak D, Atanasijevic T, Koretsky AP, Latour LL and McGavern DB: Transcranial amelioration of inflammation and cell death after brain injury. Nature 505: 223-228, 2014.

12. Warriner RA III and Hopf HW: The effect of hyperbaric oxygen in the enhancement of healing in selected problem wounds. Undersea Hyperb Med 39: 923-935, 2012.

13. Palzur E, Vlodavsky E, Mulla H, Arieli R, Feinsod M and Soustiel JF: Hyperbaric oxygen therapy for reduction of secondary brain damage in head injury: An animal model of brain contusion. J Neurotrauma 21: 41-48, 2004.

14. Harch PG, Kriedt C, Van Meter KW and Sutherland RJ: Hyperbaric oxygen therapy improves spatial learning and memory in a rat model of chronic traumatic brain injury. Brain Res 1174: 120-129, 2007.

15. Vlodavsky E, Palzur E and Soustiel JF: Hyperbaric oxygen therapy reduces neuroinflammation and expression of matrix metalloproteinase-9 in the rat model of traumatic brain injury. Neuropathol Appl Neurobiol 32: 40-50, 2006.

16. Palzur E, Zaaroor M, Vlodavsky E, Milman F and Soustiel JF: Neuroprotective effect of hyperbaric oxygen therapy in brain injury is mediated by preservation of mitochondrial membrane properties. Brain Res 1221: 126-133, 2008.

17. Teasdale G and Jennett B: Assessment of coma and impaired consciousness. A practical scale. Lancet 2: 81-84, 1974.

18. Bennett MH, Trytko B and Jonker B: Hyperbaric oxygen therapy for the adjunctive treatment of traumatic brain injury. Cochrane Database Syst Rev 12: CD004609, 2012.

19. Tal S, Hadanny A, Berkovitz N, Sasson E, Ben-Jacob E and Efrati S: Hyperbaric oxygen may induce angiogenesis in patients suffering from prolonged post-concussion syndromedue to traumatic brain injury. Restor Neurol Neurosci 33: 943-951, 2015.

20. Moher D, Liberati A, Tetzlaff J and Altman DG; PRISMA Group: Preferred reporting items for systematic reviews and meta-analyses: The PRISMA Statement. Int J Surg 8: 336-341, 2010.

21. Higgins JP and Green S: Cochrane Handbook for Systematic Reviews of Interventions: Cochrane Book Series. John Wiley and Sons, Ltd., Chichester, UK, 2008. doi: 10.1002/9780470712184. ch1.

22. Bennett M: Hyperbaric oxygen therapy no better than sham in improving post-concussion symptoms following mild traumatic brain injury. Diving Hyperb Med 43: 173, 2013.

23. Efrati $\mathrm{S}$ and Ben-Jacob E: Reflections on the neurotherapeutic effects of hyperbaric oxygen. Expert Rev Neurother 14: 233-236, 2014.

24. Davis MC, Shoja MM, Tubbs SR and Griessenauer CJ: Hyperbaric oxygen therapy for chronic post-concussive syndrome. Med Gas Res 4: 8, 2014.

25. Harch PG: Hyperbaric oxygen in chronic traumatic brain injury: Oxygen, pressure, and gene therapy. Med Gas Res 5: 9, 2015.

26. Hoge $\mathrm{C}$ and Jonas WB: The ritual of hyperbaric oxygen and lessons for the treatment of persistent postconcussion symptoms in military personnel. JAMA Intern Med 175: 53-54, 2015. 
27. Mychaskiw G: Known knowns, known unknowns and unknown unknowns: The science and the passion of $\mathrm{HBO} 2$ therapy and traumatic brain injury: An editorial perspective. Undersea Hyperb Med 40: 371-372, 2013.

28. Brkic P, Sanja P, Danijela K and Jovanović T: Hyperbaric oxygenation as an adjuvant therapy for traumatic brain injury: A review of literature. Periodicum Biologorum 116: 29-36, 2014.

29. Figueroa $X A$ and Wright JK: Clinical results in brain injury trials using HBO2 therapy: Another perspective. Undersea Hyperb Med 42: 333-351, 2015.

30. Guedes VA, Song S, Provenzano M and Borlongan CV: Understanding the pathology and treatment of traumatic brain injury and posttraumatic stress disorder: A therapeutic role for hyperbaric oxygen therapy. Expert Rev Neurother 16: 61-70, 2016.

31. Hu Q, Manaenko A, Guo Z, Huang L, Tang J and Zhang JH: Hyperbaric oxygen therapy for post concussion symptoms: Issues may affect the results. Med Gas Res 5: 10, 2015.

32. McCrary BF, Weaver L, Marrs K, Miller RS, Dicks C, Deru K, Close N and DeJong M: Hyperbaric oxygen (HBO) for post-concussive syndrome/chronic TBI product summary. Undersea Hyperb Med 40: 443-467, 2013.

33. Mitchell SJ and Bennett MH: Unestablished indications for hyperbaric oxygen therapy. Diving Hyperb Med 44: 228-234, 2014.

34. Canadian Agency for Drugs and Technologies in Health (CADTH): Hyperbaric Oxygen Therapy for Adults with Mental Illness: A Review of the Clinical Effectiveness. In: CADTH Rapid Response Reports. CADTH, Ottawa, ON, Canada, 2014.

35. Armistead-Jehle P and Lee D: Response to the Harch Group's 'A phase I study of low-pressure hyperbaric oxygen therapy for blast-induced post-concussion syndrome and post-traumatic stress disorder'. J Neurotrauma 29: 2513-2515, 2012.

36. Harch PG, Andrews SR, Fogarty EF, Amen D, Pezzullo JC, Lucarini J, Aubrey C, Taylor DV, Staab PK and Van Meter KW: Response to the letter to the editor by Armistead-Jehle and Lee on Harch et al., "A Phase I Study of Low-Pressure Hyperbaric Oxygen Therapy for Blast-Induced Post-Concussion Syndrome and Post-Traumatic Stress Disorder". J Neurotrauma 29: 2516-2519, 2012

37. Harch PG: Department of defense trials for hyperbaric oxygen and TBI: Issues of study design and questionable conclusions. Undersea Hyperb Med 40: 469-470, 2013.

38. Hoge CW and Jonas WB: Hyperbaric oxygen treatment for persistent postconcussion symptoms-reply. JAMA Intern Med 175: 1241, 2015.

39. Marois P, Mukherjee A and Ballaz L: Hyperbaric oxygen treatment for persistent postconcussion symptoms-A Placebo effect? JAMA Intern Med 175: 1239-1240, 2015.

40. Miller RS, Weaver LK and Brenner LA: Hyperbaric oxygen treatment for persistent postconcussion symptoms-reply. JAMA Intern Med 175: 1240-1241, 2015.

41. Weaver LK, Cifu D, Hart B, Wolf G and Miller RS: Reply: Department of Defense trials for hyperbaric oxygen and TBI Issues of study design and questionable conclusions. Undersea Hyperb Med 40: 471-472, 2013.

42. Wolf EG, Baugh LM, Kabban CM, Richards MF and Prye J: Cognitive function in a traumatic brain injury hyperbaric oxygen randomized trial. Undersea Hyperb Med 42: 313-332, 2015.

43. Wortzel HS, Arciniegas DB, Anderson CA, Vanderploeg RD and Brenner LA: A phase I study of low-pressure hyperbaric oxygen therapy for blast-induced post-concussion syndrome and post-traumatic stress disorder: A neuropsychiatric perspective. J Neurotrauma 29: 2421-2424, 2012.

44. Miller RS, Brenner L, Churchill S, et al: A phase II, randomized, sham-controlled trial hyperbaric oxygen for post-concussion syndrome: Impact of intervention on symptoms and well being. J Neurotrauma 30: A56, 2013.

45. Walker W, Cifu D, West S, Sima A; Graham C, Hart B, Franke LM and Carne W: Hyperbaric oxygen for blast related post-concussion syndrome: 3 -month outcomes. Brain Injury 28 668, 2014.

46. Hu Q, Liang X, Chen D, Chen Y, Doycheva D, Tang J, Tang J and Zhang JH: Delayed hyperbaric oxygen therapy promotes neurogenesis through reactive oxygen species/hypoxia-inducible factor- $1 \alpha / \beta$-catenin pathway in middle cerebral artery occlusion rats. Stroke 45: 1807-1814, 2014

47. Wolf EG, Prye J, Michaelson R, Brower G, Profenna L and Boneta O: Hyperbaric side effects in a traumatic brain injury randomized clinical trial. Undersea Hyperb Med 39: 1075-1082, 2012
48. Deng J, Lei C, Chen Y, Fang Z, Yang Q, Zhang H, Cai M, Shi L, Dong $\mathrm{H}$ and Xiong L: Neuroprotective gases-fantasy or reality for clinical use? Prog Neurobiol 115: 210-245, 2014.

49. Eovaldi B and Zanetti C: Hyperbaric oxygen ameliorates worsening signs and symptoms of post-traumatic stress disorder. Neuropsychiatr Dis Treat 6: 785-789, 2010.

50. Morries LD, Cassano P and Henderson TA: Treatments for traumatic brain injury with emphasis on transcranial near-infrared laser phototherapy. Neuropsychiatr Dis Treat 11: 2159-2175, 2015.

51. Raji CA, Tarzwell R, Pavel D, Schneider H, Uszler M, Thornton J, van Lierop M, Cohen P, Amen DG and Henderson T: Clinical utility of spect neuroimaging in the diagnosis and treatment of traumatic brain injury: A systematic review. PLoS One 9: e91088, 2014

52. Wright JK, Zant E, Groom K, Schlegel RE and Gilliland K: Case report: Treatment of mild traumatic brain injury with hyperbaric oxygen. Undersea Hyperb Med 36: 391-399, 2009.

53. Cifu DX, Walker WC, West SL, Hart BB, Franke LM, Sima A, Graham CW and Carne W: Hyperbaric oxygen for blast-related postconcussion syndrome: Three-month outcomes. Ann Neurol 75: 277-286, 2014.

54. Cifu DX, Hoke KW, Wetzel PA, Wares JR, Gitchel G and Carne W: Effects of hyperbaric oxygen on eye tracking abnormalities in males after mild traumatic brain injury. J Rehabil Res Dev 51: 1047-1056, 2014.

55. Cifu DX, Hart BB, West SL, Walker W and Carne W: The effect of hyperbaric oxygen on persistent postconcussion symptoms. J Head Trauma Rehabil 29: 11-20, 2014

56. Boussi-Gross R, Golan H, Fishlev G, Bechor Y, Volkov O, Bergan J, Friedman M, Hoofien D, Shlamkovitch N, Ben-Jacob E and Efrati S: Hyperbaric oxygen therapy can improve post concussion syndrome years after mild traumatic brain injury-randomized prospective trial. PLoS One 8: e79995, 2013.

57. Harch PG: Hyperbaric oxygen therapy for post-concussion syndrome: Contradictory conclusions from a study mischaracterized as sham-controlled. J Neurotrauma 30: 1995-1999, 2013.

58. Eyres S, Carey A, Gilworth G, Neumann V and Tennant A: Construct validity and reliability of the Rivermead Post-Concussion symptoms questionnaire. Clin Rehabil 19: 878-887, 2005.

59. Bliese PD, Wright KM, Adler AB, Cabrera O, Castro CA and Hoge CW: Validating the primary care posttraumatic stress disorder screen and the posttraumatic stress disorder checklist with soldiers returning from combat. J Consult Clin Psychol 76: 272-281, 2008

60. Rice VJ, Overby C, Boykin G, Jeter A and Villarreal J: How do I handle my life now? Coping and the post traumatic stress disorder checklist-military version. Proc Hum Factors Ergonomics Soc Ann Meet 58: 1252-1256, 2014.

61. Gardner PJ, Knittel-Keren D and Gomez M: The posttraumatic stress disorder checklist as a screening measure for posttraumatic stressdisorder in rehabilitation after burn injuries. Arch Phys Med Rehabil 93: 623-628, 2012.

62. King PR, Donnelly KT, Donnelly JP, Dunnam M, Warner G, Kittleson CJ, Bradshaw CB, Alt M and Meier ST: Psychometric study of the neurobehavioral symptom inventory. J Rehabil Res Dev 49: 879-888, 2012.

63. Ibarra S: Immediate post-concussion assessment and cognitive testing. In: Encyclopedia of Clinical Neuropsychology. Kreutzer JS, DeLuca J and Caplan B (eds). Springer, New York, pp1297-1299, 2011.

64. Kashluba S, Paniak C, Blake T, Reynolds S, Toller-Lobe G and Nagy J: A longitudinal, controlled study of patient complaints following treated mild traumatic brain injury. Arch Clin Neuropsychol 19: 805-816, 2004.

65. Bohnen N, Jolles J and Twijnstra A: Neuropsychological deficits in patients with persistent symptoms six months after mild head injury. Neurosurgery 30: 692-696, 1992.

66. Bazarian JJ, McClung J, Shah MN, Cheng YT, Flesher W and Kraus J: Mild traumatic brain injury in the United States, 1998-2000. Brain Inj 19: 85-91, 2005.

67. Al Sayegh A, Sandford D and Carson AJ: Psychological approaches to treatment of postconcussion syndrome: A systematic review. J Neurol Neurosurg Psychiatry 81: 1128-1134, 2010.

68. Mulkey DK, Henderson RA III, Putnam RW and Dean JB: Pressure $(<$ or $=4$ ATA) increases membrane conductance and firing rate in the rat solitary complex. J Appl Physiol (1985) 95: 922-930, 2003 
69. Gabizon I, Shiyovich A, Novack V, Khalameizer V, Yosefy C, Moses SW and Katz A: Impact of descent and stay at a Dead sea resort (low altitude) on patients with systolic congestive heart failure and an implantable cardioverter defibrillator. Isr Med Assoc J 13: 402-407, 2011.

70. Goldbart AD, Cohen AD, Weitzman D and Tal A: Effects of rehabilitation winter camps at the Dead Sea on European cystic fibrosis patients. Isr Med Assoc J 9: 806-809, 2007.

71. Falk B, Nini A, Zigel L, Yahav Y, Aviram M, Rivlin J, Bentur L, Avital A, Dotan R and Blau H: Effect of low altitude at the Dead Sea on exercise capacity and cardiopulmonary response to exercise in cystic fibrosis patients with moderate to severe lung disease. Pediatr Pulmonol 41: 234-241, 2006.

72. Collet JP, Vanasse M, Marois P, Amar M, Goldberg J, Lambert J, Lassonde M, Hardy P, Fortin J, Tremblay SD, et al: Hyperbaric oxygen for children with cerebral palsy: A randomised multicentre trial. HBO-CP Research Group. Lancet 357: 582-586, 2001.

73. Sham Accessed September 13, 2013. Available from: URL: http://www.merriam-webster.com/medical/sham.

74. Potter S, Leigh E, Wade D and Fleminger S: The rivermead post concussion symptoms questionnaire: A confirmatory factor analysis. J Neurol 253: 1603-1614, 2006.

75. Lin JW, Tsai JT, Lee LM, Lin CM, Hung CC, Hung KS, Chen WY, Wei L, Ko CP, Su YK and Chiu WT: Effect of hyperbaric oxygen on patients with traumatic brain injury. Acta Neurochir Suppl 101: 145-149, 2008.

76. Mychaskiw G II and Stephens PL: Hyperbaric oxygen, mild traumatic brain injury, and study design: An elusive target. J Neurotrauma 30: 1681-1682, 2013.

77. Peskind ER, Brody D, Cernak I, McKee A and Ruff RL: Military- and sports-related mild traumatic brain injury: Clinical presentation, management, and long-termconsequences. J Clin Psychiatry 74: 180-188, 2013.
78. Liu XH, Zhao YL, Ma QM, Zhou XH and Wang Y: Optimal therapeutic window of hyperbaric oxygenation in neonatal rat with hypoxic-ischemic brain damage. Zhonghua Er Ke Za Zhi 44: 177-181, 2006 (In Chinese).

79. Liu XH, Yan H, Xu M, Zhao YL, Li LM, Zhou XH, Wang MX and Ma L: Hyperbaric oxygenation reduces long term brain injury and ameliorates behavioral function by suppression of apoptosis in a rat model of neonatal hypoxia-ischemia. Neurochem Int 62: 922-930, 2013

80. Zhu M, Lu M, Li QJ, Zhang Z, Wu ZZ, Li J, Qian L, $\mathrm{Xu} \mathrm{Y}$ and Wang ZY: Hyperbaric oxygen suppresses hypoxic-ischemic brain damage in newborn rats. J Child Neurol 30: 75-82, 2015.

81. Scorza KA, McCarthy W, Miller RS, Carne W and Wolf G: Hyperbaric oxygen effects on PTSD and mild TBI symptoms: A subset analysis. Undersea Hyperb Med (UHMS Annual Meeting abstracts) 5: 1066-2936, 2013. http://archive.rubicon-foundation. org/10677.

82. Hooker JS: Hyperbaric oxygen therapy: Using evidence-based medicine to heal injured brain tissue. NC Med J 77: 69-70, 2016.

This work is licensed under a Creative Commons Attribution-NonCommercial-NoDerivatives 4.0 International (CC BY-NC-ND 4.0) License. 